American Journal of Biochemistry and Biotechnology 8 (1): 38-43, 2012

ISSN 1553-3468

(C) 2012 Science Publications

\title{
Antibiotic Compounds from Bacillus: Why are they so Amazing?
}

\author{
Patrick Fickers \\ Unit of Biotechnology and Bioprocess, \\ Universite libre de Bruxelles, Av. F.-D. Roosevelt 50, \\ CP165/61 B-1050 Brussels, Belgium
}

\begin{abstract}
The constant increase of multi-drug resistant pathogens stimulates research, more than ever, to identify and develop new antibacterial compounds. The recent advances in genome sequencing have highlighted the genus Bacillus as an unexpected source of antibiotic-like compounds. This review focus on the different class of antimicrobial molecules produce by Bacillus genus such as polyketides, nonribosomal peptide, bacteriocins as well as other unusual peptides.
\end{abstract}

Key words: Bacillus, polyketides, nonribosomal peptides, bacteriocins, lantibiotics

\section{INTRODUCTION}

The discovery of penicillin by A. Fleming more than 90 years ago has revolutionized the medicine and allowed to treat millions of people from bacterial infections. Unfortunately, the intensive use and misuse of antibiotics have generated a strong selective pressure for the emergence of resistant strains. Actually, the constant increase of multi-drug resistant pathogenic microorganisms stim ulates more than ever effort to identify and develop new antibacterial compounds. In that field, the recent advances in genome sequencing have highlighted the genus Bacillus as an unexpected source of antibiotic-like compounds. Indeed, for some of them, such as Bacillus subtilis, more than $4 \%$ of the genome has been found potentially devoted to the synthesis of Polyketides (PKs), Nonribosomal Peptide (NRPs), bacteriocins as well as other unusual antibiotics (Kunst et al., 1997; Chen et al., 2009a; Arguelles-Arias et al., 2009). In the past, these compounds had to be identified by intensive screening for antimicrobial activity against appropriate targets and subsequently purified using fastidious methods prior to assess their potential utilization as antibacterial or antifungal compound. However, since the advent of the genomic era, available bacterial genomes are screened for bacteriocin, Polyketide Synthetase (PKS) and Nonribosomal Peptide-Synthetase (NRPS) gene clusters using appropriate bioinformatics tools, rendering the identification of new compounds more easy-going (Tapi et al., 2010; Caboche et al., 2008).

Polyketides and non-ribosomal peptide: Polyketides (PKs) and Nonribosomal Peptides (NRPs) are synthesized by large multimodular synthetase by elongation of activated monomers of amino and hydroxyl acid building blocks. The genes coding for these synthetases are clustered in operons than can span over $150 \mathrm{~kb}$. NRPSs are organized in modules responsible for the incorporation of a specific amino acid. The modules consist of three main core domains that catalyze a specific reaction for the incorporation of a monomer. Firstly, the adenylation domain (A) selects and activates the cognate amino acid as an amino acyladenylate. Then after, Thiolation domain (T) covalently binds the activated monomer to the synthetase through a phosphopantetheinyl arm. Finaly, the condensation domain (C) catalyses the formation of the peptide linkage between the activated amino acids from two adjacent $\mathrm{T}$ modules. Two additional specific modules are also requested for NRPs synthesis. These are the loading module, composed only by one $\mathrm{A}$ and $\mathrm{T}$ domain, involved in the activation and incorporation of the first amino-acid of the NRP and a termination module containing a Thioesterase (TE) domain, which releases the newly synthetized peptide from the synthetase. Beside these three main domains, many NRPSs feature more specialized domains within modules that allow residue modifications, namely Epimerisation (E), Methylation (M), Oxidation (Ox), Reduction (R), Formylation (F) and Heterocyclisation (Cy). These specialized domains enable NRPSs to synthesize an impressive number of diversified structures with broad range of biological activities that could not be obtained by the ribosomal machinery (Felnagle et al., 2008). This diversity of structure is responsible for their board-range of biological activities, targets, mechanisms of action, allowing their exploitation by the biopharmaceutical industry. Bacitracin, for instance, used in human medicine, inhibit the biosynthesis of the bacterial cell wall by interacting with undecaprenyl pyrophosphate, involved in peptidoglycan synthesis (Stone and Strominger, 1971). Since its US FDA approval in 2010, bacitracin is used for the treatment of infants with staphylococcal pneumonia and empyema. Mechanistically, NRPS could function based on two distinct schemes. Linear NRPSs proceed synthesis starting with the loading module, following by addition of specific amino acid according the ordered fashion of the different modules 
in the synthetase and ending with the cleavage of the peptide from the synthetase by the TE domain. Iterative NRPSs use successively, one, several or the entire set of modules constituting the synthetases (Mootz et al., 2002). Since the discovery of gramicidin from $B$. brevis, the first NRP compound characterized in the early '70, many bioactive NRPs have been isolated and their biosynthetic clusters characterized. Recently, the Norine database containing hundreds of NRPS molecules has been created, providing users with an interesting computational tool for systematic study of these molecules (Caboche et al., 2008). With emergence of powerful bioinformatics and molecular tools, identifying new metabolites by genome mining has become a reality (Ansari et al., 2004; Lanen and Shen, 2006). For instance, a bioinformatics analysis of the genome of $B$. thuringiensis led to the prediction of an NRPS constituted of seven modules that could be involved in the synthesis of a heptalipopeptide similar to kurstakin (Abderrahmani et al., 2011). The discovery of new natural products by genome mining is an encouraging sign, suggesting that this methodology could lead to the isolation of novel molecules of pharmacological interest.

PKSs assemble the core structure of polyketides from acyl-Coenzyme A (acyl-CoA) monomers in a head-to-tail fashion (Jenke-Kodama and Dittmann, 2009; Hertweck et al., 2007). Type I PKSs are similar to NRPSs in that the different catalytic domains are found in a single polypeptide and are further subdivided into iterative and modular synthases. An iterative type I PKS is a monomodular synthase in which a single set of catalytic domains is used repeatedly in a highly programmed fashion. By contrast, modular PKSs feature several separate modules that do not repeat. Type II PKSs possess a biosynthetic mechanism analogous to iterative type I PKSs but harbour their catalytic domains on mono-or bi-functional proteins (Hertweck et al., 2007). Several bacteria, including $B$. subtilis and fungi, but mostly plants, possess type III PKSs (Nakano et al., 2009; Resmi and Soniya, 2012; Li et al., 2011). They consist of a single multimodular protein synthesizing molecules devoid of any antibimicrobial activities (Felnagle et al., 2008). Each PKS module is composed by at least three core domains: an Acyltransferase Domain (AT) which selects the appropriate monomers, an acyl Carrier Protein Domain (ACP) that carry the activated monomer through the formation of a thioester linkage and a Ketosynthase (KS) domain responsible for the condensation between the activated monomer and the polyketide intermediate present on two adjacent ACP domains. Additional secondary domains such as Ketoreductase (KR), Oxidation (Ox), Dehydratase (DH), Methyltransferase (MT), Enoylreductase (ER) and Methylation (M) domains are involved in the chemical modification of the growing polyketide. Type
II PKSs often feature a Cyclase (Cy) domain leading to the formation of aromatic structures. Similarly to NRPS, the last module contains a Thioesterase (TE) domain that catalyzes the release of the final product from the enzyme (Meurer et al., 1997). Due to their versatile assemblage mechanism, polyketides exhibit remarkable diversity both in terms of structure and biological activities. For instance, three functional gene clusters directing the synthesis of difficidin, macrolactin and bacillaene were identified in $B$. amyloliquefaciens (Arguelles-Arias et al., 2009; Chen et al., 2007). Difficidin is an unsaturated 22-membered macrocylic polyene lactone phosphate ester with broadspectrum antibacterial activity (Wilson et al., 1987; Zimmerman et al., 1987). It inhibits protein biosynthesis and was recently shown promising in its suppressive action against Erwinia amylovara, a devasting plant pathogen causing necrotrophic fire blight disease of apple, pear and other rosaceous plants (Zweerink and Edison, 1987; Chen et al., 2009b). By contrast, macrolactin and bacillaene have not yet been demonstrated to be directly related to biocontrol, although they are both antimicrobial agents that could be potentially useful in human medicine. Macrolactin, which consists of a 24-membered ring lactone, had the ability to inhibit murine melanoma cancer cells as well as mammalian herpes simplex viruses. It was also shown effective in protecting lymphoblast cells from HIV (Gustafson et al., 1989). Similarly to difficidin, bacillaene is an inhibitor of prokaryotic protein synthesis constituted by an open-chain enamine acid with an extended polyene system. This compound displays antimicrobial activity toward human pathogens such as Serratia marcescens, Klebsiella pneumoniae and Staphylococcus aureus (Patel et al., 1995).

NRPS/PKS hybrid synthetases: Several compounds isolated from bacteria are synthesized by NRPS/PKS hybrid synthetases. These metabolites are composed of a polyketide backbone featuring incorporated amino acids in the case of a PKS-NRPS hybrid or a peptidyl chain harbouring ketone group characteristic of an NRPS-PKS hybrid. Among them are the three iturin lipopeptides, namely mycosubtilin (Duitman et al., 1999), iturin A (Tsuge et al., 2001) and bacillomycin D (Hofemeister et al., 2004), produced by different strain of Bacillus. Iturins are amphiphilic cyclic peptides composed of seven $\alpha$-amino acids linked to a single $\beta$ amino fatty acid. The length of the fatty acid moiety may vary from $\mathrm{C} 14$ to $\mathrm{C} 17$ and different homologous compounds with a linear or branched fatty acid moiety are usually co-produced (Hofemeister et al., 2004). This amphiphilic structure confers interesting biological properties to these secondary metabolites. For instance, mycosubtilin, produced by B. subtlis ATCC6633, was found very effective against the human pathogenic 
yeast Candida albicans (Fickers et al., 2008; 2009) and as biocontrol agent to prevent Pythium infections of tomato seedlings (Leclere et al., 2005). The gene clusters involved in mycosubtilin is composed of one operon that span over $38 \mathrm{~kb}$. It consists of four ORFs, designated $f e n F, m y c A, \quad m y c B$ and $m y c C$. The mycosubtilin synthetase reveals features unique for peptide synthetases as well as for fatty acid synthases. MycA subunit combines functional domains derived from peptide synthetases, amino transferases and fatty acid synthases. The growing understanding of PKS and NRPS mechanisms has led to the development of genetic engineering technique yielding to the synthesis of "synthetic" compounds. These methods consist of adding, subtracting or permuting modules or catalytic domains of a known natural synthase to obtain NRPs and PKs presenting modified structures and functions (Cane et al., 1998).

Bacteriocin: Beside NRP and PK molecules, Bacillus genus is also able to produce interesting ribosomally synthetised peptides, the so-called bacteriocin (Lee and Kim, 2011; Abriouel et al., 2011). Based on their structure and biological activities, bacteriocins could be divided into three main groups. Class I bacteriocins, the so-called lantibiotics, are characterized by their unusual amino acids such as Lanthionine (Lan), Methyllanthionine (MeLAn) and dehydrated residues. Lan and MeLan are enzymatically synthesized by the cyclisation of a free cysteine and a dehydrated residue, namely Dehydroalanine (Dha) and didehydrobutyrine (Dhb), respectively. DhA and Dhb are obtained by the dehydratation of a serine or a threonine (McAuliffe et al., 2001). Lantibiotics can be further subdivided into two types based on their structure and mode of posttranslational modifications. Type A lantibiotics exhibit linear secondary structures and are positively charged. Two distinct LanB and LanC enzymes are involved in their post-translational modification and they are processed by a LanP protease. By contrast, Type B lantibiotics, exhibit a globular structure and are non-charged. They are modified by a single LanM enzyme and processed by a LanT transporter with $\mathrm{N}$ terminal-associated protease activity (Willey and Donk, 2007). Beside this, two-component lantibiotics, consisting of a two peptides system that acts synergistically were also reported. Since a single LanM enzyme modifies these peptides, they were classified as type B lantibiotics (Asaduzzaman and Sonomoto, 2009). Compared to lantibiotics, class II bacteriocins are non-modified peptides that are synthesized ribosomally. They are characterized by a molecular weigh below $5 \mathrm{kDa}$. They could be further subdivided into three subclasses (IIa, IIb, IIc) based on structural properties, activity and mode of action (Nes and Tagg, 1996; Klaenhammer, 1993). Class IIa bacteriocins are characterized by an hydrophobic N-termini containing the YGNGV consensus sequence and a disulfide bridge. Class IIb feature two-component non-modified bacteriocin whereas class IIc regroups all the other molecules that do not correspond to class IIa and IIb. Class III bacteriocins are heat sensitive molecules with a molecular weight higher than $30 \mathrm{kDa}$. Its only representative among the Bacillus genera is the megacin A-216 produced by $B$. megaterium that only exhibits a phospholipase A2 activity (Tersch and Carlton, 1984). The type IA bacteriocin subtilin, produced by $B$. subtilis ATCC6633, is the model lantibiotic produced by members of the Bacillus genus. Active subtilin contains eight post-transcriptionally modified residues: four methyllanthionines (Abu-SAla), one didehydrobutyrine, one dehydroalanine and one lanthionine (Ala-S-Ala) and one D-alanine (Banerjee and Hansen, 1988). Despite their relative variability of structure, lantibiotics share in common some characteristic features concerning their mode of synthesis. The gene cluster involved in their synthesis, which is approximately $10-15 \mathrm{~kb}$, is composed of a structural gene as well as other genes necessary for the modification, transport, regulation and immunity of the producer strain. The subtilin biosyntheric gene cluster is composed of 10 ORFs, namely spaBTCSIFEGRK, forming four distinct operons (spaBTC, spaS, spaIFEG, spaRK). SpaS encodes a premature and inactive peptide of 56 residues with a 24 residues signal sequence. SpaB and $\mathrm{SpaC}$ are involved in the dehydratation of serine and threonine, which are required for the formation of Dha and Dhb, respectively, as well as that of lanthionine, which is the result of the cyclisation of Dha with thiol group of a free cysteine. Presubtilin is exported by the ABC type transporter protein SpaT and is then processed by the serine protease subtilisin to form a bioactive subtilin. Self-protection of the producer strain from the synthesized lantibiotics is ensured by different immunity mechanisms. The protein SpaI, a membrane-bound protein, interacts specifically with subtilin causing its inactivation. Beside this, the immunity complex formed by SpaF, SpaE and SpaG proteins pumps subtilin out of the producer strain. The production of lantibiotic is regulated at the transcriptional level in a cell-densitydependent manner. It was evidenced that some lantibiotic, such as subtilin, can act as auto-inducing peptides (Kleerebezem et al., 2004). Two proteins, spaR and spaK, corresponding to a response regulator protein and a sensor kinase, respectively, form the regulatory system. After subtilin concentration in the culture medium reaches a certain threshold, it activates the membrane-bound SpaK protein which then autophosphorylates. This leads to the phosphorylation of SpaR, which in phosphorylated form can recognize the binding domain on three promoters upstream of 
spas, spaBTC and spaIFEG, resulting in subtilin production. In addition, SpaRK expression is controlled by the sporulation transcription factor sigma $\mathrm{H}$ (Stein $e t$ al., 2002). All of these highlight that lantibiotics synthesis is correlated to both to cell density and to sporulation mechanism. The biological activity of lantibiotics is centered on Gram-positive bacteria and consists of pores formation in target cell membranes and/or inhibition of peptidoglycane synthesis by interacting with undecaprenylpyrophosphorylMurNAc-(pentapeptide)-GlcNAc, the so-called lipid II (McAuliffe et al., 2001; Bierbaum and Sahl, 2009). For some lantibiotics, such as epidermin, lipid II serve as a docking molecule that facilitate the pore formation (Parisot et al., 2008; Brotz and Sahl, 2000).

Unusual peptide: Bacillus sp were also found able to synthetize others unusual antibiotic peptides. Among them, rhizocticins are phosphonate oligopeptide antibiotics containing the C-terminal nonproteinogenic amino acid (Z)-1-2-Amino-5-Phosphono-3-Pentenoic Acid (APPA) (Borisova et al., 2010; Kino, 2010; Kino et al., 2009). The molecules are synthesized by the socalled L-amino acid ligase. These enzymes are able to catalyze the formation of an alpha-peptide bond from L-amino acids in an ATP-dependent manner. For instance, YwfE from B. subtilis 168, was the first reported L-amino acid ligase able to synthesizes various dipeptides (Tabata et al., 2005). Rhizocticin A, is known to inhibit the growth of yeast and filamentous fungi but is not active against bacteria. Rhizocticins enters the target cell via the oligopeptide transport system and must be cleaved by a peptidase in order to release the active APPA, which inhibit threonine synthase, an enzyme that catalyse the Pyridoxal 5'Phosphate (PLP)-dependent conversion of phosphohomoserine to L-threonine (Kino et al., 2009; Laber et al., 1994; Diddens et al., 1979). Hence, APPA interferes with threonine synthesis ultimately affecting protein synthesis and leading to growth inhibition (Borisova et al., 2010). Beside APPA based antibacterial compounds, some strains of B. subtilis produce the dipeptide bacilysin composed of L-alanine and the unusual amino acid L-anticapsin (Walker and Abraham, 1970). Anticapsin is able to inhibit glucosamine synthetize and thus causes a failure in the bacterial wall synthesis and finaly the growth of bacteria (Kenig et al., 1976).

\section{CONCLUSION}

The amazing biological properties of de different type of Bacillus's compounds described in this review could be one answer to the multi-drug resistant concerns. To date, it is estimated that only a small fraction of the antimicrobial molecules potentially produced by Gram-positive bacteria has been identified. Research to discover these compounds is sure to be ongoing for many more years.

\section{REFERENCES}

Abderrahmani, A., A. Tapi, F. Nateche, M. Chollet and V. Leclere et al., 2011. Bioinformatics and molecular approaches to detect NRPS genes involved in the biosynthesis of kurstakin from Bacillus thuringiensis. Applied Microbiol. Biotechnol., 92: 571-581. DOI: 10.1007/s00253011-3453-6

Abriouel, H., C.M.A.P. Franz, N.B. Omar and A. Galvez, 2011. Diversity and applications of Bacillus bacteriocins. FEMS Microbiol. Rev., 35: 201-232. DOI: 10.1111/j.1574-6976.2010.00244.x

Ansari, M.Z., G. Yadav, R.S. Gokhale and D. Mohanty, 2004. NRPS-PKS: A knowledge-based resource for analysis of NRPS/PKS megasynthases. Nucleic Acids Res., 32: W405-W413. DOI: 10.1093/nar/gkh359

Arguelles-Arias, A., M. Ongena, B. Halimi, Y. Lara and A. Brans et al., 2009. Bacillus amyloliquefaciens GA1 as a source of potent antibiotics and other secondary metabolites for biocontrol of plant pathogens. Microb. Cell Fact., 8: 63-63. DOI: $10.1186 / 1475-2859-8-63$

Asaduzzaman, S.M. and S. Sonomoto, 2009. Lantibiotics: diverse activities and unique modes of action. J. Biosci. Bioeng., 107: 475-487. DOI: 10.1016/j.jbiosc.2009.01.003

Banerjee, S. and J.N. Hansen, 1988. Structure and expression of a gene encoding the precursor of subtilin, a small protein antibiotic. J. Biol. Chem., 263: 9508-9514.

Bierbaum, G. and H.G. Sahl, 2009. Lantibiotics: Mode of action, biosynthesis and bioengineering. Curr. Pharm. Biotechnol., 10: 2-18. DOI: 10.2174/138920109787048616

Borisova, S.A., B.T. Circello, J.K. Zhang, W.A.V.D. Donk and W.W. Metcalf, 2010. Biosynthesis of rhizocticins, antifungal phosphonate oligopeptides produced by Bacillus subtilis ATCC6633. Chem. Biol., 17: 28-37. DOI: 10.1016/j.chembiol.2009.11.017

Brotz, H. and H.G. Sahl, 2000. New insights into the mechanism of action of lantibiotics-diverse biological effects by binding to the same molecular target. J. Antimicrob. Chemother., 46: 1-6. DOI: 10.1093/jac/46.1.1

Caboche, S., M. Pupin, V. Leclere, A. Fontaine and P. Jacques et al., 2008. NORINE: A database of nonribosomal peptides. Nucl. Acids Res., 36: D326-D331. DOI: 10.1093/nar/gkm792 
Cane, D.E., C.T. Walsh and C. Khosla, 1998. Harnessing the biosynthetic code: Combinations, permutations and mutations. Science, 282: 63-68. DOI: $10.1126 /$ science. 282.5386 .63

Chen, X.H., A. Koumoutsi, R. Scholz, A. Eisenreich and K. Schneider et al., 2007. Comparative analysis of the complete genome sequence of the plant growth-promoting bacterium Bacillus amyloliquefaciens FZB42. Nat. Biotechnol., 25: 1007-1014. DOI: $10.1038 /$ nbt1325

Chen, X.H., A. Koumoutsi, R. Scholz and R. Borriss, 2009a. More than Anticipated - Production of Antibiotics and Other Secondary Metabolites by Bacillus amyloliquefaciens FZB42. J. Mol. Microbiol. Biotechnol., 16: 14-24. DOI: 10.1159/000142891

Chen, X.H., A. Koumoutsi, R. Scholz, K. Schneider and J. Vater et al., 2009b. Genome analysis of Bacillus amyloliquefaciens FZB42 reveals its potential for biocontrol of plant pathogens. J. Biotechnol., 140: 27-37. DOI: 10.1016/j.jbiotec.2008.10.011

Diddens, H., M. Dorgerloh and H. Zahner, 1979. Metabolic products of microorganisms 176. On the transport of small peptide antibiotics in bacteria. J. Antibiot., 32 : 87-90. DOI: 10.7164/antibiotics.32.87

Duitman, E.H., L.W. Hamoen, M. Rembold, G. Venema and H. Seitz et al., 1999. The mycosubtilin synthetase of Bacillus subtilis ATCC6633: A multifunctional hybrid between a peptide synthetase, an amino transferase and a fatty acid synthase. Proc. Natl. Acad. Sci. USA., 96: 13294-13299. DOI: 10.1073/pnas.96.23.13294

Felnagle, E.A., E.E. Jackson, Y.A. Chan, A.M. Podevels and A.D. Berti et al., 2008. Nonribosomal peptide synthetases involved in the production of medically relevant natural products. Mol. Pharm., 5: 191-211. DOI: 10.1021/mp700137g

Fickers, P., V. Leclere, J.S. Guez, M. Bechet and F. Coucheney et al., 2008. Temperature dependence of mycosubtilin homologue production in Bacillus subtilis ATCC6633. Res. Microbiol., 159: 449-457. DOI: 10.1016/j.resmic.2008.05.004

Fickers, P., J.S. Guez, C. Damblon, V. Leclere and M. Béchet et al., 2009. High-Level Biosynthesis of the Anteiso-C17 Isoform of the Antibiotic Mycosubtilin in Bacillus subtilis and Characterization of Its Candidacidal Activity. Appl. Environ. Microbiol., 75: 4636-4640. DOI: 10.1128/AEM.00548-09

Gustafson, K.R., J.H. Cardellina, R.W. Fuller, O.S. Weislow and R.F. Kiser et al., 1989. AIDSantiviral sulfolipids from cyanobacteria (blue-green algae). J. Natl. Cancer Inst., 81: 1254-1258. DOI: 10.1093/jnci/81.16.1254
Hertweck, C., A. Luzhetskyy, Y. Rebets and A. Bechthold, 2007. Type II polyketide synthases: Gaining a deeper insight into enzymatic teamwork. Nat. Prod. Rep., 24: 162-90. DOI: 10.1039/B507395M

Hofemeister, J., B. Conrad, B. Adler, B. Hofemeister and J. Feesche et al., 2004. Genetic analysis of the biosynthesis of non-ribosomal peptide- and polyketide-like antibiotics, iron uptake and biofilm formation by Bacillus subtilis A1/3. Mol. Genet. Genomics., 272: 363-378. DOI: 10.1007/s00438004-1056-y

Jenke-Kodama, H. and E. Dittmann, 2009. Evolution of metabolic diversity: Insights from microbial polyketide synthases. Phytochemistry, 70: 18581868. DOI: 10.1016/j.phytochem.2009.05.021

Kenig, M., E. Vandamme and E.P. Abraham, 1976. The Mode of Action of Bacilysin and Anticapsin and Biochemical Properties of Bacilysin-resistant Mutants. J. Gen. Microbiol., 94: 46-54. DOI: 10.1099/00221287-94-1-46

Kino, K., Y. Kotanaka, T. Arai and M. Yagasaki, 2009. A Novel L-amino acid ligase from bacillus subtilis NBRC3134, a microorganism producing peptideantibiotic rhizocticin. Biosci. Biotechnol. Biochem., 73: 901-907. DOI: 10.1271/bbb. 80842

Kino, K., 2010. Novel L-amino acid ligases catalyzing oligopeptide synthesis. Yakugaku Zasshi., 130: 1463-1469. DOI: 10.1248/yakushi.130.1463

Klaenhammer, T.R., 1993. Genetics of bacteriocins produced by lactic acid bacteria. FEMS Microbiol. Rev., 12: 39-85. DOI: 10.1111/j.15746976.1993.tb00012.x

Kleerebezem, M., R. Bongers, G. Rutten, W.D. Vos and O. Kuipers, 2004. Autoregulation of subtilin biosynthesis in Bacillus subtilis: The role of the spa-box in subtilin-responsive promoters. Peptides., 25: 1415-1424. DOI: 10.1016/j.peptides.2003.11.025

Kunst, F., N. Ogasawara, I. Moszer, A.M. Albertini and G. Alloni et al., 1997. The complete genome sequence of the gram-positive bacterium Bacillus subtilis. Nature, 390: 249-256. DOI: $10.1038 / 36786$

Laber, B., K.P. Gerbling, C. Harde, K.H. Neff and E. Nordhoff et al., 1994. Mechanisms of interaction of Escherichia coli threonine synthase with substrates and inhibitors. Biochemistry, 33: 341323. DOI: $10.1021 / \mathrm{bi00177a035}$

Lanen, S.G.V. and B. Chen, 2006. Microbial genomics for the improvement of natural product discovery. Curr. Opin. Microbiol., 9: 252-260. DOI: 10.1016/j.mib.2006.04.002 
Leclere, V., M. Bechet, A. Adam, J.S. Guez and B. Wathelet et al., 2005. Mycosubtilin overproduction by Bacillus subtilis BBG100 enhances the organism's antagonistic and biocontrol activities. Applied Environ. Microbiol., 71: 4577-4584. DOI: 10.1128/AEM.71.8.4577-4584.2005

Lee, H. and H. Kim, 2011. Lantibiotics, class I bacteriocins from the genus Bacillus. J. Microbiol. Biotechnol., 21: 229-235. PMID: 21464591

Li, J., Y. Luo, J.K. Lee and H. Zhao, 2011. Cloning and characterization of a type III polyketide synthase from Aspergillus niger. Bioorg. Med. Chem. Lett., 21: 6085-6089. DOI: 10.1016/j.bmcl.2011.08.058

McAuliffe, O., R.P. Ross and C. Hill, 2001. Lantibiotics: Structure, biosynthesis and mode of action. FEMS Microbiol. Rev., 25: 285-308. DOI: 10.1016/S0168-6445(00)00065-6

Meurer, G., M. Gerlitz, E. Wendt-Pienkowski, L.C. Vining and J. Rohr et al., 1997. Iterative type II polyketide synthases, cyclases and ketoreductases exhibit context-dependent behavior in the biosynthesis of linear and angular decapolyketides. Chem. Biol., 4: 433-443. DOI: 10.1016/S10745521(97)90195-2

Mootz, H.D., D. Schwarzer and M.A. Marahiel, 2002. Ways of assembling complex natural products on modular nonribosomal peptide synthetases. Chem. Bio. Chem., 3: 490-504. DOI: 10.1002/14397633(20020603)3:6<490::AIDCBIC490>3.0.CO;2-N

Nakano, C., H. Ozawa, G. Akanuma, N. Funa and S. Horinouchi, 2009. Biosynthesis of aliphatic polyketides by type III polyketide synthase and methyltransferase in Bacillus subtilis. J. Bacteriol., 191: 4916-4923. DOI: 10.1128/JB.00407-09

Nes, I.F. and J.R. Tagg, 1996. Novel lantibiotics and their pre-peptides. Ant. Van Leeuwenhoek, 69: 8997. DOI: 10.1007/BF00399414

Parisot, J., S. Carey, E. Breukink, W.C. Chan and A. Narbad et al., 2008. Molecular mechanism of target recognition by subtilin, a class I lanthionine antibiotic. Antimicrob. Agent. Chemother., 52: 612-618. DOI: 10.1128/AAC.00836-07

Patel, P., S. Huang, S. Fisher, D. Pirnik and C. Aklonis et al., 1995. Bacillaene, a Novel Inhibitor of Procaryotic Protein Synthesis Produced by Bacillus subtilis: Production, taxonomy, isolation, physicochemical characterization and biological activity. J. Antibiot., 48: 997-1003. DOI: 10.7164/antibiotics.48.997

Resmi, M.S. and E.V. Soniya, 2012. Molecular cloning and differential expressions of two cDNA encoding Type III polyketide synthase in different tissues of Curcuma longa L. Gene, 491: 278-283. PMID: 21986037
Stein, T., S. Borchert, P. Kiesau, S. Heinzmann and S. Kloss et al., 2002. Dual control of subtilin biosynthesis and immunity in Bacillus subtilis. Mol. Microbiol., 44: 403-416. DOI: 10.1046/j.1365-2958.2002.02869.x

Stone, K.J. and J.L. Strominger, 1971. Mechanism of action of bacitracin: Complexation with metal ion and $\mathrm{C}_{55}$-Isoprenyl pyrophosphate. Proc. Natl. Acad. Sci. U SA., 68: 3223-3227. DOI: 10.1073/pnas.68.12.3223

Tabata, K., H. Ikeda and S.I. Hashimoto, 2005. ywfE in Bacillus subtilis codes for a novel enzyme, Lamino acid ligase. J. Bacteriol., 187: 5195-5202. DOI: $10.1128 / J B .187 .15 .5195-5202.2005$

Tapi, A., M. Chollet-Imbert, B. Scherens and P. Jacques, 2010. New approach for the detection of non-ribosomal peptide synthetase genes in Bacillus strains by polymerase chain reaction. Applied Microbiol. Biotechnol., 85: 1521-1531. DOI: 10.1007/s00253-009-2176-4

Tersch, M.A.V. and B.C. Carlton, 1984. Molecular cloning of structural and immunity genes for megacins A-216 and A-19213 in Bacillus megaterium. J. Bacteriol., 160: 854-859.

Tsuge, K., T. Akiyama and M. Shoda, 2001. Cloning, Sequencing and characterization of the iturin a operon. J. Bacteriol., 183: 6265-6273. DOI: 10.1128/JB.183.21.6265-6273.2001

Walker, J.E. and E.P. Abraham, 1970. The structure of bacilysin and other products of Bacillus subtillis. Biochem. J., 118: 563-570. PMCID: PMC1179252

Willey, J.M. and W.A.V.D. Donk, 2007. Lantibiotics: Peptides of diverse structure and function. Ann. Rev. Microbiol., 61: 477-501. DOI: 10.1146/annurev.micro.61.080706.093501

Wilson, K.E., J.E. Flor, R.E. Schwartz, H. Joshua and J.L. Smith et al., 1987. Difficidin and oxydifficidin: novel broad spectrum antibacterial antibiotics produced by bacillus subtilis II. isolation and physico-chemical characterization. J. Antibiot., 40: 1682-1691. DOI: 10.7164/antibiotics.40.1682

Zimmerman, S.B., C.D. Schwartz, R.L. Monaghan, B.A. Pelak and B. Weissberger et al., 1987. Difficidin and oxydifficidin: Novel broad spectrum antibacterial antibiotics produced by Bacillus subtilis. I. Production, taxonomy and antibacterial activity. J. Antibiot., 40: 1677-1681. DOI: 10.7164/antibiotics.40.1677

Zweerink, M.M. and A. Edison, 1987. Difficidin and oxydifficidin: novel broad spectrum antibacterial antibiotics produced by Bacillus subtilis. III. Mode of action of difficidin. J. Antibiot., 40: 1692-1697. DOI: 10.7164/antibiotics.40.1692 